\title{
Disminución del desarrollo de cáncer experimental del colon en ratas alimentadas con leche de búfala*
}

\author{
Ramírez, G.V.; Villordo, G.; Montenegro, M.A.; Catuogno, M.S.; Sánchez Negrette, M. \\ Cátedra de Patología General y Sistemática, Facultad de Ciencias Veterinarias, Universidad Nacional del \\ Nordeste, Sargento Cabral 2139, Corrientes (3400), Argentina. Tel/Fax: 03783-425753. \\ Email: patgral@vet.unne.edu.ar.
}

\begin{abstract}
Resumen
Ramírez, G.V.; Villordo, G.; Montenegro, M.A.; Catuogno, M.S.; Sánchez Negrette, M.: Disminución del desarrollo de cáncer experimental del colon en ratas alimentadas con leche de búfala. Rev. vet. 23: 1, 15-19, 2012. La leche bovina contiene elementos potencialmente capaces de inhibir la carcinogénesis; a su vez, la leche bubalina tiene un alto valor debido a su mayor concentración de sólidos totales, grasa, proteína y lactosa. El objetivo del presente trabajo consistió en determinar el efecto anticancerígeno de la leche bubalina sobre el desarrollo de tumores y focos de criptas displásicas (FCD) en colon de ratas tratadas con el cancerígeno 1,2-dimetilhidrazina. Las ratas $(n=78)$ fueron divididas al azar en lotes que recibieron agua o leche, con y sin tratamiento con el cancerígeno, durante 123 y 240 días. Al final de la experiencia se realizó en cada animal el análisis macro y microscópico del intestino grueso, determinándose presencia, número y ubicación de tumores y de FCD. Los resultados demostraron disminución significativa del número de tumores $(60 \%)$ y de FCD $(62,5 \%)$ en los lotes que recibieron leche al compararlos con los que recibieron agua. Estos resultados permiten demostrar que la leche de búfala disminuye tanto el desarrollo de tumores como de lesiones displásicas de colon en un modelo de carcinogénesis experimental del intestino grueso.
\end{abstract}

Palabras clave: búfalo, leche, cáncer de colon, 1,2-dimetilhidrazina.

\begin{abstract}
Ramírez, G.V.; Villordo, G.; Montenegro, M.A.; Catuogno, M.S.; Sánchez Negrette, M.: Reduced development of experimental colon cancer in rats fed with buffalo milk. Rev. vet. 23: 1, 15-19, 2012. Bovine milk contains elements with potential to inhibit carcinogenesis; in turn, buffalo milk is of a high value as it contains more total solids, fat, protein and lactose compared to the former. The objective of this study was to determine the anticancer effect of buffalo milk on the development of tumors and dysplastic crypt foci (DCF) in colon of rats treated with the carcinogen 1,2-dimethylhydrazine. Rats $(\mathrm{n}=78)$ were randomly divided into lots that received water or milk, with and without treatment with the carcinogen, during 123 and 240 days. At the end of the experiment macro and microscopic analysis of the large intestine were performed on each animal, determining the presence, number and location of tumors and DCF. The results showed significant reduction in tumor number $(60 \%)$ and DCF $(62.5 \%)$ in lots that received milk compared to those that received water. These results allow us to demonstrate that buffalo milk reduces both the development of tumors and dysplastic lesions of colon carcinogenesis in an experimental model of the large intestine.
\end{abstract}

Key words: buffalo, milk, colon cancer, 1,2-dimethylhydrazine.

\section{INTRODUCCIÓN}

Desde la década de 1970 la incidencia de cáncer de colon se ha incrementado en los países occidentales y se ha convertido en la segunda/tercera causa de muerte por cáncer, tanto en hombres como mujeres ${ }^{5,13}$. Su etiología es compleja e implica factores genéticos y

Recibido: 18 octubre 2011 / Aceptado: 2 diciembre 2011

*Proyecto subsidiado por la Agencia Nacional de Promoción

Científica y Tecnológica PICTO-UNNE 119. ambientales; entre estos últimos, los hábitos alimenticios juegan un rol preponderante ${ }^{21}$. La relación entre el cáncer de colon y componentes de la dieta, tales como grasas, fibras, vegetales, vitaminas y otros elementos, han sido evaluados en estudios epidemiológicos, así como en trabajos experimentales. Estudios epidemiológicos en diferentes poblaciones y experimentales en animales de laboratorio, mantienen la hipótesis de que la cantidad y tipo de grasa de la dieta y composición de los ácidos grasos son factores determinantes en la carcinogénesis del colon. Trabajos realizados en seres 
humanos relacionan el cáncer intestinal, principalmente el cáncer colorrectal, con la ingestión de alimentos ricos en grasa de origen animal y escaso consumo de fibras ${ }^{21}$.

Si bien la grasa de los productos bovinos (leche y carne) en muchos casos es considerada perjudicial para la salud por su alto contenido en ácidos grasos saturados, en los últimos años se ha encontrado que varios componentes de la leche tienen propiedades anticancerígenas, además de actividad lipolítica, preventiva de la arteriosclerosis y la diabetes, por lo que se la considera un alimento funcional ${ }^{14}$. Tales componentes son: esfingomielina, ácido linoleico conjugado (ALC), ácido butírico, vitaminas A y D, entre otros ${ }^{18}$. A su vez, numerosos estudios han determinado que la leche bubalina también tiene un alto valor, debido a que posee más sólidos totales, grasa, proteína y lactosa que la leche bovina ${ }^{19}$.

El objetivo del presente trabajo consistió en determinar el efecto anticancerígeno de la leche bubalina sobre el desarrollo de tumores y focos de criptas displásicas (FCD) en el colon de ratas tratadas con el cancerígeno 1,2-dimetilhidrazina (DMH).

\section{MATERIAL Y MÉTODOS}

Se utilizaron 78 ratas Wistar machos, con edad aproximada de 6 semanas ( $160 \mathrm{~g})$, que fueron alojadas en un ambiente climatizado con temperatura controla$\mathrm{da}\left(21^{\circ} \mathrm{C}\right)$, mantenidas en jaulas metálicas individuales, con agua y dieta de alimento balanceado ad-libitum. Los animales fueron divididos de manera aleatoria en dos grupos: 1 (controles) y 2 (experimentales, inoculados con DMH). A su vez, cada uno de estos grupos se dividió en lotes, tal como se explicita a continuación.

Grupo 1 (animales controles, $\mathrm{n}=16$ ): compuesto por cuatro lotes, a saber: lote 1 : cuatro ratas con dieta y agua ad-libitum (123 días), lote 2: cuatro ratas con dieta y leche ad-libitum (123 días), lote 3: cuatro ratas con dieta y agua ad-libitum (240 días) y lote 4: cuatro ratas con dieta y leche ad-libitum (240 días).

Grupo 2 (animales experimentales, inoculados con $\mathrm{DMH})$, alojados en un recinto separado del grupo control, compuesto por cuatro lotes, a saber: lote 5: quince ratas con dieta y agua ad-libitum + DMH (123 días), lote 6: quince ratas con dieta y leche ad-libitum $+\mathrm{DMH}$ (123 días), lote 7: seis ratas con dieta y agua ad-libitum + DMH (240 días) y lote 8: seis ratas con dieta y leche ad-libitum + DMH (240 días).

La leche se obtuvo de 8 búfalas multíparas de razas Murrah y mestizas Murrah x Mediterránea, alimentadas con pasturas naturales, mayoritariamente por especies como Andropogon lateralis, A. sellononaus, Cynodon dactylon, Elionorus sp, Paspalum notatum, P. almun chase, Sorghastrum agrostoides, Desmodium canum y Shylosanthes macrosona. Previamente a la administración de la dieta láctea a las ratas, se tomaron muestras de leche a todas las búfalas a fin de determinar su contenido en ácidos grasos. La administración de leche a las ratas comenzó una semana antes de la primera inoculación con DMH. Los tumores intestinales fueron inducidos en los lotes experimentales del grupo 2, mediante una inyección subcutánea semanal de DMH durante 5 semanas, a la dosis de $20 \mathrm{mg} / \mathrm{kg}$ de peso corporal. La solución de DMH fue preparada con $400 \mathrm{mg}$ de la droga disuelta en $100 \mathrm{ml}$ de agua destilada estéril, conteniendo $37 \mathrm{mg}$ de EDTA como agente estabilizador y mantenida a pH 6,5 usando hidróxido de sodio. Esta solución fue preparada cada semana, momentos antes de cada inoculación.

Al final de la experiencia todas las ratas fueron sacrificadas. En todos los animales, el intestino grueso fue extraído en su totalidad, abierto longitudinalmente, colocado sobre una plancha de cartón y fijado con formol bufferado al $10 \%$ durante 24 horas.

Para el estudio macroscópico se determinó el número, tamaño y localización de los tumores en los diferentes segmentos anatómicos del intestino grueso (recto, colon distal, colon proximal y ciego). Los tumores fueron clasificados según su aspecto macroscópico en polipoides, con sus variedades pediculados y de base sésil, y no polipoides, con sus variedades plano con aspecto de placa y ulceroinfiltrantes. Para el estudio del tamaño, los tumores fueron agrupados en 5 categorías: de 1 a 5 , de 6 a 10 , de 11 a 15 , de 16 a 20 y más de 20 $\mathrm{mm}$.

Para el estudio microscópico las muestras fueron procesadas con la técnica clásica para bloques parafinados, cortadas en micrótomo tipo Minot en $5 \mu \mathrm{m}$ de espesor y coloreadas con la técnica de hematoxilinaeosina (HyE). Cortes de cada uno de los segmentos del intestino grueso fueron examinados a fin de detectar, localizar y caracterizar los FCD. Los tumores fueron clasificados en adenomas tubulares (AT), adenomas tubulovellosos (ATV), adenocarcinomas simples (ACS), carcinomas de células en anillo de sello (Ccas), carcinomas mucinosos $(\mathrm{Cmu})$ y carcinomas mixtos (Cmix).

\section{RESULTADOS}

Las ratas consumieron la leche de búfala sin manifestar ningún tipo de rechazo. Todos los animales se adaptaron a las condiciones del bioterio y fueron ganando peso a lo largo de la experiencia, observándose que las ratas que consumieron leche terminaron con un peso mayor en relación a las que consumieron solamente agua, tanto a los 123 días (pesos promedio lote 1: 425 g. vs lote 2: $452,5 \mathrm{~g}$ y lote $3: 472,5 \mathrm{~g}$ vs lote $4: 480 \mathrm{~g}$ ) como a los 240 días (pesos promedio lote 5: $454 \mathrm{~g}$ vs lote 6: 464,66 g, lote 7: $415 \mathrm{~g}$ vs lote 8: $430 \mathrm{~g}$ ). No se observaron alteraciones físicas ni cambios del comportamiento en ninguna de las ratas tratadas con la droga cancerígena.

La Tabla 1 muestra que al final de la experiencia, las necropsias revelaron disminución del número de tumores $(60 \%)$ en las ratas que recibieron leche de búfala durante 123 días (lote 6: dos tumores), al compararlas con las ratas que sólo recibieron agua (lote 5: cinco tu- 
Tabla 1. Localización de tumores en los 4 lotes experimentales del grupo 2 .

\begin{tabular}{lcccc}
\hline localización & $\begin{array}{c}\text { lote } 5 \\
\text { (DMH+agua) }\end{array}$ & $\begin{array}{c}\text { lote } 6 \\
(\mathrm{DMH}+\text { leche) }\end{array}$ & $\begin{array}{c}\text { lote } 7 \\
(\mathrm{DMH}+\text { agua) }\end{array}$ & $\begin{array}{c}\text { lote } 8 \\
(\mathrm{DMH}+\text { leche })\end{array}$ \\
\hline recto & 0 & 0 & 0 & 0 \\
colon distal & 4 & 2 & 8 & 3 \\
colon proximal & 1 & 0 & 1 & 0 \\
ciego & 0 & 0 & 0 & 1 \\
tumores totales & 5 & 2 & 9 & 4 \\
\hline
\end{tabular}

Tabla 2. Localización de focos de criptas displásicas (FCD) en los 4 lotes del grupo 2 .

\begin{tabular}{lcccc}
\hline localización & $\begin{array}{c}\text { lote } 5 \\
\text { (DMH+agua) }\end{array}$ & $\begin{array}{c}\text { lote } 6 \\
\text { (DMH+leche) }\end{array}$ & $\begin{array}{c}\text { lote } 7 \\
(\mathrm{DMH}+\text { agua) }\end{array}$ & $\begin{array}{c}\text { lote } 8 \\
(\mathrm{DMH}+\text { leche) }\end{array}$ \\
\hline recto & 0 & 0 & 0 & 0 \\
colon distal & 16 & 4 & 8 & 3 \\
colon proximal & 7 & 5 & 1 & 1 \\
ciego & 1 & 0 & 0 & 0 \\
FCD totales & 24 & 9 & 9 & 4 \\
\hline
\end{tabular}

mores). Las ratas del lote 8 que recibieron leche durante 240 días, también redujeron el número de tumores (55,56\%: cuatro tumores) al compararlas con el lote 7 que sólo recibió agua (nueve tumores).

En los 4 lotes experimentales del grupo 2, la mayor frecuencia de tumores correspondió al tipo polipoide sésil (Figura 1), y en segundo lugar al tipo plano. En ningún animal se constataron tumores ulceroinfiltrantes ni polipoides pediculados.

El colon distal fue asiento de la mayor concentración de tumores, seguido por el colon proximal y el ciego, no encontrándose tumores en recto, sin diferencias importantes entre ambos períodos de la experimentación. En cuanto al tamaño de los tumores, en los lotes 5 y 6 se mantuvieron en el rango de 1 a $5 \mathrm{~mm}$, mientras que en el lote 7 se observó un tumor en el rango de 11$15 \mathrm{~mm}$ y en el lote 8 un tumor en el rango de $6-10 \mathrm{~mm}$. Los tipos histológicos encontrados correspondieron en su totalidad a neoplasias malignas, con un total de 17 ACS, 2 Ccas y 1 Cmix en los cuatro lotes experimentales.

La Tabla 2 señala que los FCD mostraron una considerable disminución en todos los animales del grupo experimental alimentados con leche. Se registró disminución del $62,5 \%$ en el lote 6 comparado con el lote 5 , que solo consumió agua (9 vs 24). En el grupo experimental 2 también se observó disminución del número de FCD del $55,56 \%$ en el lote 8 con respecto al lote 7 (4 vs 9). La frecuencia de FCD fue mayor en el colon distal (Figura 2), seguido por el colon proximal, no encontrándose $\mathrm{FCD}$ en el recto.

Los resultados obtenidos demuestran que en ambos períodos de experimentación, la leche de búfala disminuyó substancialmente tanto el desarrollo de tumores como el de focos de criptas displásicas en este modelo de carcinogénesis experimental del intestino grueso.

\section{DISCUSIÓN}

Varios estudios han demostrado que la leche de vaca y sus componentes, tales como esfingomielina, ácido butírico, ácido linoleico conjugado (ALC) y vitaminas A y D, entre otros ${ }^{21}$, ejercen efectos inhibitorios sobre el desarrollo de tumores en varios modelos de carcinogénesis experimental en ratas y ratones.

La esfingomielina es el fosfolípido que predominantemente reside en la membrana externa de muchos tejidos animales y cuya función comprende la regulación de la fluidez de membrana. Su concentración y la de otros fosfolípidos son del 0,2 a $1 \%$ del total de lípidos de la leche ${ }^{4}$, asociados con la membrana del glóbulo graso. Se ha demostrado que la ceramida y la esfingosina participan en tres de las vías anti-proliferativas de regulación celular: inhibición del crecimiento celular, inducción de la diferenciación e inducción de apoptosis o muerte ce-

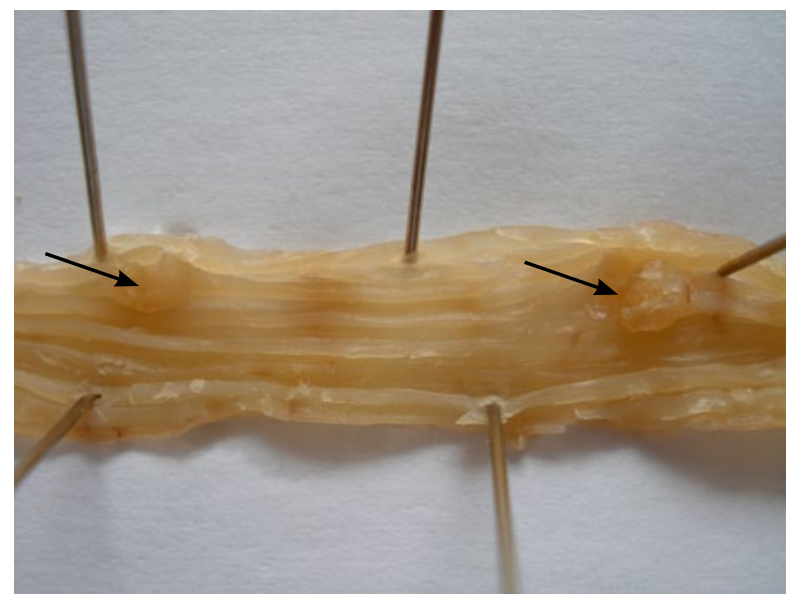

Figura 1. Intestino grueso de rata del Lote 5 con dos tumores de tipo polipoide sésil en colon distal (flechas).

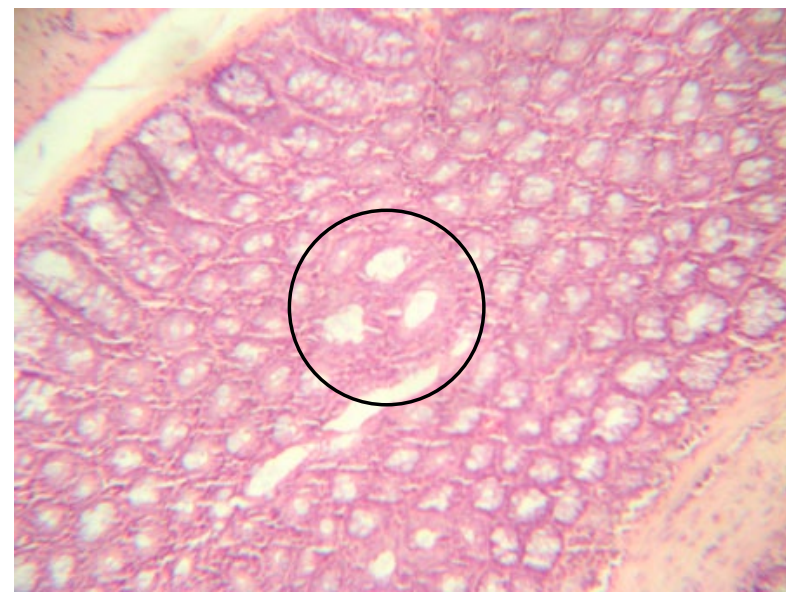

Figura 2. FCD con displasia leve (círculo) que compromete la parte media de la mucosa, correspondiente a un segmento de colon distal de una rata del lote 7 (HyE $10 \mathrm{x}$ ). 
lular programada. Estas vías pueden contribuir a la supresión de la oncogénesis. Los metabolitos bioactivos de esfingomielina, ceramida y esfingosina, pueden ser referidos como lípidos supresores de tumores ${ }^{8}$. Las investigaciones sugieren que la absorción y el metabolismo de la esfingomielina a través del tracto gastrointestinal puede proteger al órgano de la carcinogénesis ${ }^{11,23}$.

El ácido butírico es un inhibidor de la proliferación $\mathrm{y}$ un potente inductor de la diferenciación in vitro para una gran variedad de células neoplásicas, incluyendo células de leucemia, linfoma, mama, colon, recto, hígado, cérvix, ovario y tejido nervioso ${ }^{3}$. También puede producir efectos de maduración en células no cancerosas, modular la expresión de oncogenes y suprimir genes en varios tipos de células. Ha sido reportado que el ácido butírico no solo induce diferenciación en las líneas celulares del carcinoma colónico humano, sino que también induce apoptosis ${ }^{1,3}$ y estimula la actividad antitumoral y antiviral del interferón in vitro e in vivo ${ }^{20}$.

Uno de los componentes más importantes de la grasa láctea es el ácido linoleico conjugado (ALC), considerado un ácido graso con propiedades anticancerígenas ${ }^{17}$, antiaterogénicas e inmunomoduladoras ${ }^{18}$. En cuanto a la actividad anticancerígena del ALC, se ha demostrado reducción del número de papilomas en un modelo de carcinogénesis epidérmica en ratón ${ }^{6}$. En un segundo estudio realizado por los mismos investigadores se demostró también que el ALC disminuyó los tumores gástricos en ratas, inducidos experimentalmente con benzo(a)pyrene en cerca del $50 \%{ }^{7}$. Otros investigadores observaron disminución del número de tumores de mama inducidos experimentalmente con dimetilbenzantraceno en ratas alimentadas con ALC ${ }^{12}$.

La vitamina A y sus derivados tienen acción anticancerígena y quimiopreventiva en cultivo de células y órganos 9, 16. La deficiencia de vitamina A predispone a cambios pre-malignos y estimula el desarrollo de inductores químicos del cáncer ${ }^{16}$. Los retinoides han sido demostrados como inhibidores del estado de promoción de la tumorogénesis en piel de ratón; además, pueden actuar como agentes quimiopreventivos de cáncer epitelial de la vejiga urinaria, glándula mamaria, páncreas, cavidad oral y pulmón en animales experimentales $9,10,16$. En seres humanos, los retinoides se consideran preventivos para el cáncer de piel, cabeza, cuello, pulmón y vejiga y son efectivos en el tratamiento de la leucemia promielocítica aguda ${ }^{10}$.

En contraste con los retinoides, el rol del beta caroteno en la quimioprevención está mínimamente establecido. En animales ha sido demostrado que previene la aparición de tumores de piel inducidos por luz ultravioleta y carcinógenos ${ }^{18}$. En una experiencia en la que se administró ácido butírico durante 26 semanas a ratas Wistar, se observó en intestino grueso una marcada disminución de focos de criptas displásicas inducidas experimentalmente con el cancerígeno $\mathrm{DMH}^{2}$. También en ratas Wistar que recibieron una dieta a base de leche descremada durante 14 semanas se observó un menor número de tumores inducidos experimentalmente ${ }^{22}$.

En la presente experiencia se observó que las ratas que consumieron leche de búfala presentaron importantes disminuciones en el desarrollo de tumores y de focos de criptas displásicas. Estos resultados coinciden con otras investigaciones realizadas en las que se trabajó con dietas lácteas bovinas ${ }^{15,22}$ y permiten demostrar que la leche de búfala constituye un alimento funcional de alto valor debido a sus componentes potencialmente capaces de disminuir el proceso de la carcinogénesis en un modelo biológico de experimental.

\section{REFERENCIAS}

1. Calabrese C, Venturini L, Ronco G, Villa P, Chomienne C, Belpomme D. 1993. Butyric acid and its monosaccharide ester induce apoptosis in the HL-60 cell line. Biochem Biophys Res Comm 195: 31-38.

2. Catuogno MS, Montenegro MA, Sánchez Negrette M. 2006. Disminución del desarrollo de focos de criptas displásicas en el colon de ratas suplementadas con ácido butírico. Anales Comunic Cientif Tecnol Univ Nac Nordeste, Corrientes (Argentina), http://www.unne.edu.ar/Web/cyt/ cyt2006/04-Veterinarias/2006-V-010.pdf.

3. Chen ZX, Breitman TR. 1994. Tributyrin: A prodrug of butyric acid for potential clinical application in differentiation therapy. Cancer Res 54: 3494-3499.

4. Christie WW, Noble RC, Davies G. 1987. Phospholipids in milk and dairy products. J Soc Dairy Technol 40: 10-12.

5. Ferlay J, Bray F, Pisani P, Parkin DM. 2002. Cancer incidence, mortality and prevalence worldwide. International Agency for Research on Cancer (IARC), Cancer Base $n^{\circ}$ 5, IARC Press, Lyon. On line: http://www-dep.iarc.fr/.

6. Ha YL, Grimm NK, Pariza MW. 1987. Anticarcinogens from fried ground beef: heat-altered derivatives of linoleic acid. Carcinogenesis 8: 1881-1887.

7. Ha YL, Storkson JM, Pariza MW. 1990. Inhibition of benzo(a)pyrene-induced mouse forestomach neoplasia by conjugated dienoic derivates of linoleic acid. Cancer Res. 50: 1097-1101.

8. Hannun YA. 1994. The sphingomyelin cycle and the second messenger function of ceramide. $J$ Biol Chem 169: 3125-3128.

9. Hennekens CH, Mayrent SL, Willett W. 1986. Vitamin A, carotenoids, and retinoids. Cancer 58: 1837-1841.

10. Hill DL, Grubbs CJ. 1992. Retinoids and cancer prevention. Ann Rev Nutr 12: 161-181.

11. Imaizumi K, Tominaga A, Sato M, Sugano M. 1992. Effects of dietary sphingolipids on levels of serum and liver lipids in rats. Nutr Res 12: 543-548.

12. Ip C, Chin SF, Scimeca JA, Pariza MW. 1991. Mammary cancer prevention by conjugated dienoic derivate of linoleic acid. Cancer Res 51: 6118-6124.

13. Jemal A, Murray T, Ward E, Samuel A, Tiwari RC, Chafoor A. 2005. Cancer statistics. Cancer J Clin 55: 1030 .

14. McGuire MA, McGuire MK. 2000. Conjugated linoleic acid (CLA): A ruminant fatty acid with beneficial effects 
on human health. Proc Am Soc Anim Sci. On line: http:// www.asa.org/jas/symposia/proceedings/0938.pdf.

15. Montenegro MA, Catuogno MS, Lizarraga FD, Sánchez Negrette M. 2002. Efectos de dietas lácteas sobre la carcinogénesis experimental del intestino grueso inducida con 1, 2-dimetilhidrazina en ratas. Com Cient Tecnol Univ Nac Nord (Corrientes, Argentina). http://wwwl.unne.edu. ar/cyt/2002/04-Veterinarias/V-014.pdf.

16. Moon RC. 1989. Comparative aspects of carotenoids and retinoids as chemopreventive agents for cancer. J Nutr 119: 127-134.

17. National Research Council (NRC). 1996. Carcinogenesis and anticarcinogenesis in the human diet, National Academy Press, Washington DC, 242 p.

18. Parodi PW. 1996. Milk fat components: possible chemopreventive agents for cancer and other diseases. Australian J Dairy Tech 5: 24-32.

19. Patiño EM, Méndez FI, Faisal EL, Cedrés JF, Gómez LG, Guanziroli MC. 2003. Buffalo milk composition of Murrah and half-breed Murrah x Mediterraneo in Corrientes, Argentina. Buffalo Newsletter 18: 8-10.
20. Pouillart P, Cerutti I, Ronco G, Villa P, Chany C. 1992. Protective activity of monoacetone glucose 3-butyrate, prodrug of n-butyric acid, against the fatal effect of encephalomyocarditis virus in mice. Comptes Rendus Acad Sci III 314: 49-54.

21. Roynette EC, Calder PC, Dupertis YM, Pichard C. 2004. N-3 polyunsaturatedfatty acids and colon cancer prevention. Clin Nutr 23: 139-151.

22. Sánchez Negrette M, Montenegro MA, Lértora WJ, Catuogno MS. 2005. Disminución del número de tumores intestinales inducidos por 1,2-dimethilhidrazina en ratas alimentadas con leche descremada. Rev Vet 16: 28-31.

23. Schmelz EM, Crall KJ, Larocque R, Dillehay DL, Merrill AH. 1994. Uptake and metabolism of sphingolipids in isolated intestinal loops of mice. J Nutr 124: 702-712.

\section{Asociación Cooperadora de la Facultad de Ciencias Veterinarias Universidad Nacional del Nordeste}

Personería Jurídica No 647/92 y 912/00

Sargento Cabral 2139

3400 Corrientes

Argentina

La Asociación Cooperadora de la Facultad de Ciencias Veterinarias de la UNNE fue constituida el 10 de diciembre del año 1991 como entidad de bien público, con el objeto de promover y coadyuvar las actividades científicas, educativas y culturales relacionadas con las ciencias veterinarias. En tal sentido, implementa acciones para colaborar con la enseñanza, extensión, actualización y difusión científica que realiza dicha casa de estudios.

\section{Beneficios que brinda a sus asociados:}

- Fotocopias con descuentos especiales del $20 \%$ en la Fotocopiadora Copias.com que funciona dentro del predio de la Facultad de Ciencias Veterinarias.

- Descuentos del 10\% para la adquisición de libros de la Editorial Inter-Médica.

- Descuentos especiales en otros rubros.

Sea solidario, asóciese en beneficio de toda la comunidad universitaria

Tel. 0379-4425753 interno 186• Página Web www.vet.unne.edu.ar•E-mail: cooperadora@vet.unne.edu.ar 